\title{
Impact of External Disturbance and Discontinuous Input on the Redundant Manipulator Robot Behaviour using the Linear Parameter Varying Modelling Approach
}

\author{
Sameh Zribi \\ Research Laboratory LARA in Automatic Control, \\ ENIT, University of Tunis El Manar, \\ Tunis, Tunisia \\ Hatem Tlijani \\ Research Laboratory LARA in Automatic Control, \\ ENIT, University of Tunis El Manar, \\ Tunis, Tunisia
}

\author{
Jilani Knani \\ Research Laboratory LARA in Automatic Control, \\ ENIT, University of Tunis El Manar, \\ Tunis, Tunisia \\ Vicenç Puig \\ Department of Automatic Control (ESAII), \\ University Polytechnic of Catalonia, \\ Barcelona, Spain
}

\begin{abstract}
This paper is concerned with the synthesis of dynamic model of the redundant manipulator robot based on Linear Parameter Varying approach. To evaluate its behavior and in presence of external disturbance several motions profiles are developed using a new algorithm which produce smooth trajectories in optimal time. The main advantages of this proposed approach are its robustness and its simplicity with respect to the flexibility structure, to the motion profile and mass load variations. Numerical simulations with several tasks show that in presence of mass load variation the desired trajectory is more efficiently followed by the LPV model than the dynamic model of the studied mechanism. Its performances are ensured using the smoothest trajectory designed by the Eighth-degree polynomial profile than the Fifth-degree polynomial one and the trapezoidal one.
\end{abstract}

Keywords-Redundant manipulator robot; flexible structure; linear parameter varying approach; discontinuous torque; external disturbance

\section{INTRODUCTION}

Redundant manipulators robots, the combination of rigid and flexible structures mounted in series are of great interest in a number of applications in the modern industry [1]-[3].

Recently, the use of these manipulators containing flexible arm has received much attention. It has been an important progress several tasks like in planning [4], [5], design [6] and control [7], [8]. It gives more feasibility to robotic mechanisms because of the existence of several solutions in the specified workspace.

However, the dynamic complexity of redundant manipulator robot related to the flexible structure may cause some difficulties especially in high speeds such as external disturbance and highly nonlinearity [8]. In order to cope with this problem, we propose to apply the linear parameter varying approach to the calculated nonlinear model of the redundant manipulator robot in the first step. Then, several motions profiles are applied to evaluate the system's behaviour with a variable mass load as an external disturbance.

The contribution of this paper consists of simulating the Linear Parameter Varying (LPV) model of the considered robotic system using several inputs and evaluating the system's performances in presence of external disturbances and discontinuous inputs.

This paper is organized as follows: In Section II, the trajectory generation overview of several motions profiles is detailed. Section III deals with the presentation of the dynamic modelling of redundant manipulator mechanism followed with its LPV model. In Section IV, the simulation results testing the dynamic performance of our mechanism by applying the motions profiles are analysed with varying of its mass load. Section V presents some concluding remarks.

\section{TRAJECTORY GENERATION OVERVIEW}

In the scientific literature, almost all techniques on the problem of trajectory planning are focused on the parameter optimization [8]. The algorithms with optimized time were the first proposed techniques of trajectory generation in the literature. Moreover, some applications require the algorithms for optimized energy because of limited capacity for the energy source (for example underwater robots).

The regularity's degree of inputs will directly touch the excitation order of vibrational modes of the robotic mechanism. A trajectory containing a high and discontinuous acceleration causes during movement an important vibrational excitation of some joints of the mechanism or its entire structure [8]. In order to minimize the effector vibration and to suppress its residual vibration, the motion profile must be characterized by a limited jerk [8], [9]. So, using continuous 
acceleration, a motion profile with a limited jerk will be obtained and a smooth movement will be guaranteed. Unlike excessive jerk, its values can cause the excitation of vibrations in the mechanism structure [8].

The trajectory generation mechanism generates the reference profiles for the control system in order to ensure that the manipulator follows the planned trajectories [10]. It consists of generating a temporal sequence of the values obtained by trapezoidal and polynomial functions as the desired trajectory.

\section{A. Trapezoidal Motion Profile}

Using the mathematical principle appointed by the BangBang profile, the researcher Hermes formulated the trapezoidal motion Profile [11]. This profile consists of ensuring the saturation of the acceleration variable of control plant when its level is switched several times between its extreme values. This saturation leads to optimize the time, whereas, it needs the maximum power available to saturate the mechanism actuators [12].

In order to ensure an optimal time, the trapezoidal motion profile leads to generate a continuous speed by ensuring the saturation of both the speed and the acceleration. In the rest of this paper, a time derivation of such function is usually denoted by a number in brackets in the power of this function. The joint position of the trapezoidal profile is defined by

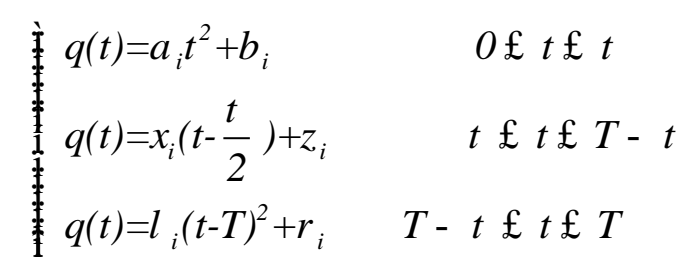

and its first derivative is given by

$$
\begin{array}{lr}
q^{(1)}(t)=2 a_{i} t & 0 £ t £ t \\
q^{(1)}(t)=x_{i} & t £ t £ T-t \\
q^{(l)}(t)=2 l_{i}(t-T) & T-t £ t £ T
\end{array}
$$

where $q(t)$ is the function according to the time of joint position, $\tau$ is the time period of the acceleration phase, $T$ is the time period of movement, $\alpha_{i}, \beta_{i}, \xi_{i}, \zeta_{i}, \lambda_{i}$ and $\rho_{i}$ are constants depending on the initial and the final positions.

Using this profile, its joint velocity is presented along the time period of 0.6 seconds as presented in Fig. 1.

The acceleration saturation produced by this profile is performed by switching between two levels. This switching causes the problem of torque discontinuity that the DC motor cannot track. Moreover, the delivered torque causes a delay in response in the real path compared to the desired path [8]. In order to remedy these problems, others motions profiles called polynomial functions will be proposed.
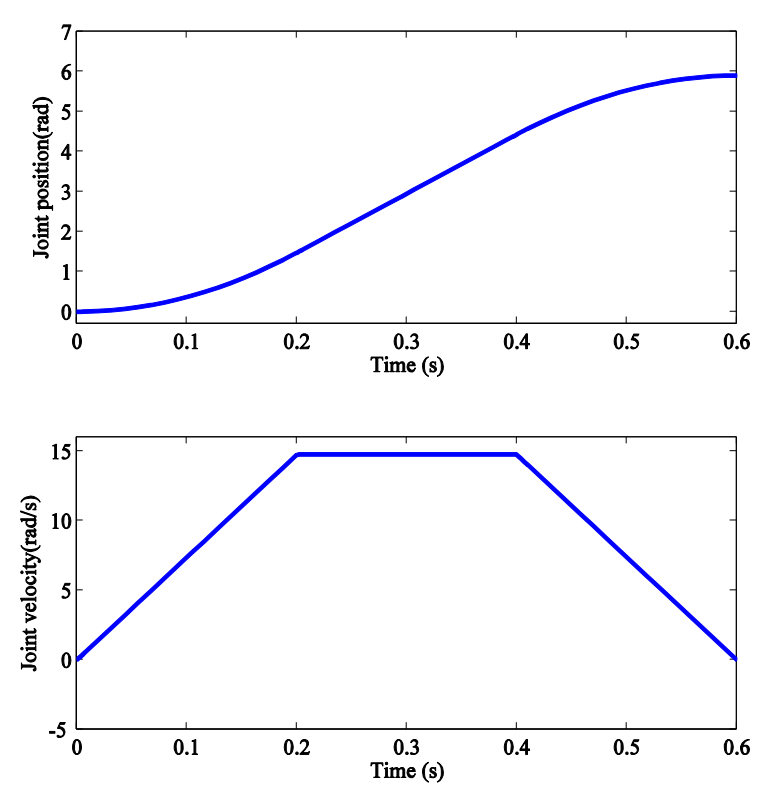

Fig. 1. Joint position and joint velocity for trapezoidal motion profile.

\section{B. Fifth-Degree Polynomial Motion Profile}

The three most common approaches are the linear, the fifth-polynomial and the eighth-polynomial interpolations functions. Its general form of these functions is expressed as follows:

$$
q(t)=q_{0}+h(t) D
$$

Where, $q_{0}$ presents the initial position, $h(t)$ presents the interpolation function and $D$ presents the difference between the final position $q_{f}$ and the initial position $q_{0}$. In this work, we limited to apply the fifth-polynomial and the eighthpolynomial interpolations functions to the studied mechanism.

Concerning the fifth-polynomial profile, it produces a smooth movement similar to that of the human movement joint. The acceleration provided by this motion profile is characterized by an excitation of natural modes and a minimum jerk, so its movement is appointed by minimum-jerk movement [13].

The joint position of the fifth-polynomial interpolation function is expressed by

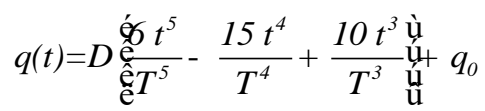

and its first derivative is given by

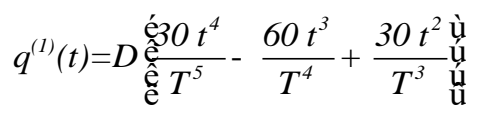

Along the time period of 0.6 seconds, the joints position and velocity of this profile are described in Fig. 2. 
In order to improve the fifth-polynomial motion profile by obtaining a smoother movement and by minimizing the response time, the eighth-polynomial function will be explained and then simulated.
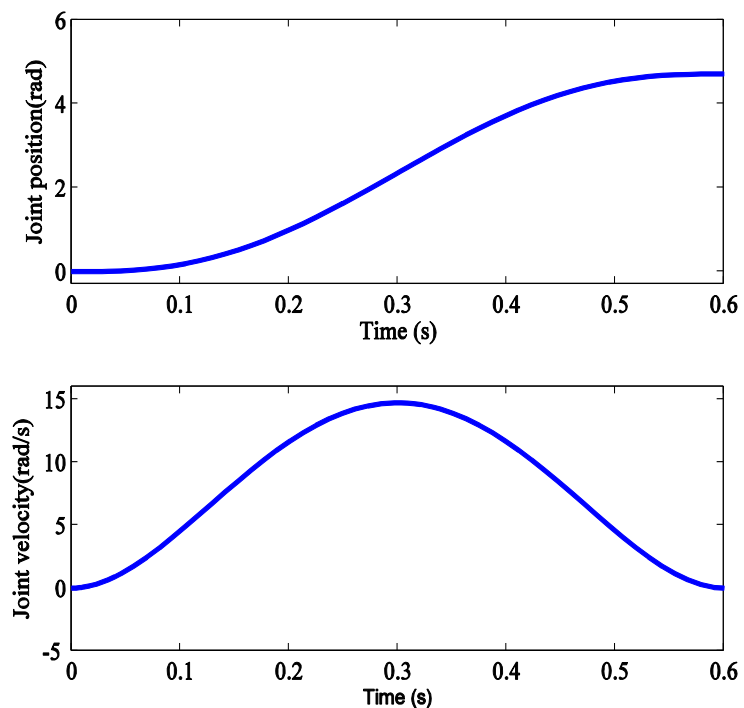

Fig. 2. Joint position and joint velocity for fifth-polynomial profile.

\section{Eighth-Degree Polynomial Motion Profile}

Smooth trajectories of given path can be generated by eighth-degree polynomial interpolation function [12]. It can be expressed by

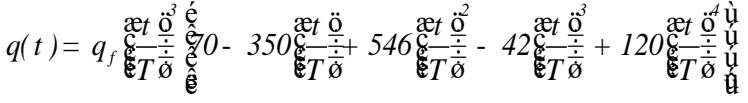

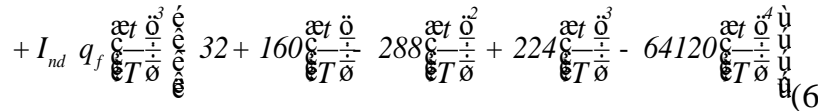

and its first derivative can be defined as follows:

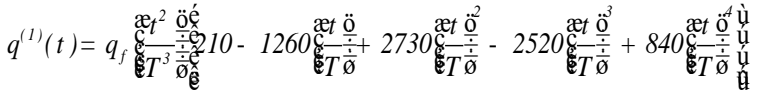

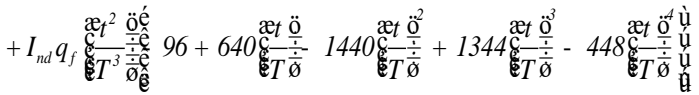

Where, $I_{n d}$ is a parameter allowing to obtain the smoothest trajectory, it is defined as follows:

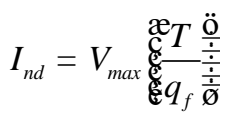

Where, $V_{\max }$ is the maximum velocity generated during the trajectory. The eighth polynomial motion profile allows issuing a smooth movement. This smoothness is ensured with this motion profile more than that with fifth-polynomial and trapezoidal motions profiles. Moreover, using the eighthpolynomial motion profile, the robustness of the system is guaranteed opposite to the external disturbances which are presented by the variation of the load mass in our work.

The joints position and velocity of this motion profile are presented along the time period of 0.6 seconds as in Fig. 3 .
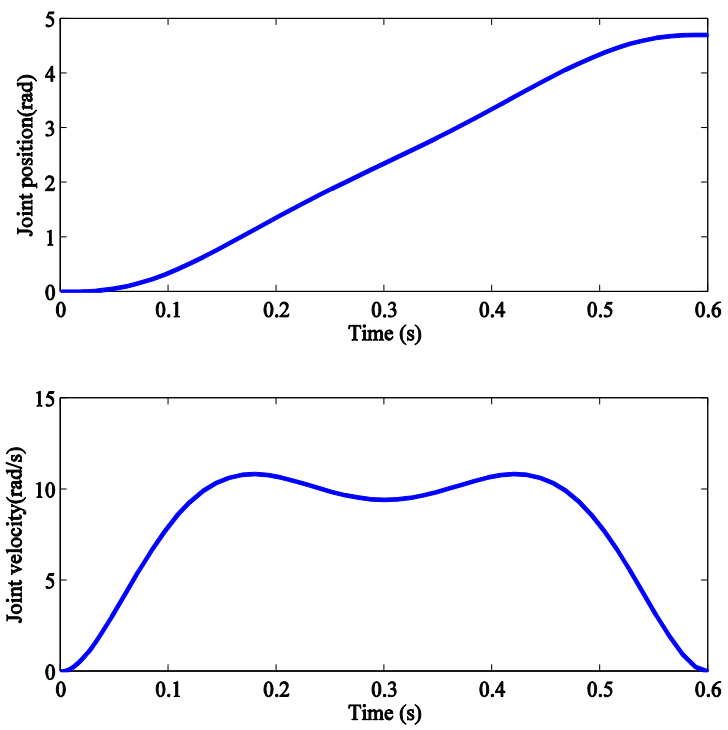

Fig. 3. Joints position and velocity for eighth-polynomial profile.

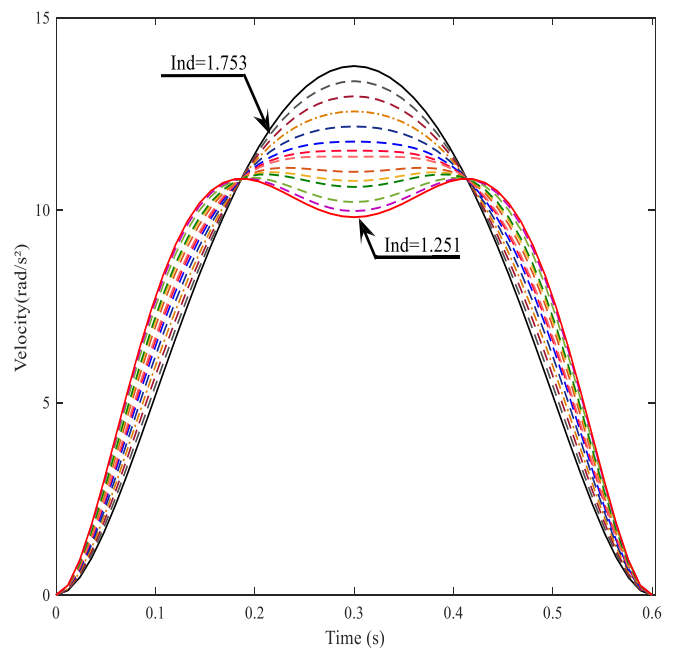

Fig. 4. Velocities profiles of the robot manipulator when $I_{n d}$ is within the interval of natural movements.

In order to generate smooth trajectories using the eighthpolynomial interpolation function, the parameter $I_{n d}$ must be in the interval [1.251, 1.753], as it is presented in Fig. 4 which shows the velocities profiles of the robot manipulator when $I_{n d}$ is within the interval of natural movements.

The use of eighth-polynomial interpolation function with variable parameter $\left(I_{n d}\right)$ allows generating a big number of smooth trajectories for a given path. If this parameter is inside 
this interval, the eighth-polynomial function generates natural movements. We can note that if $I_{n d}=1.251$, the generated slope of the velocity curve is very sharp at the beginning of the trajectory and its end, so this trajectory has the lowest $V_{\max }$ With regard to the case where $I_{n d}=1.753$, the velocity curve slope is very smooth at the beginning of the trajectory and its end, but this trajectory needs the highest $V_{\max }$ [14].

\section{LINEAR PARAMETER VARYING APPROACH FOR DYNAMIC MODELLING OF REDUNDANT MANIPULATOR ROBOT}

\section{A. Mechanism's Description and Assumptions}

Before the dynamic modelling, the redundant manipulator robot is presented by the rigid-flexible manipulator mechanism [15]. It consists of five rigid bodies and the sixth one presenting the end-effector which is considered as a flexible body. A mass load appointed by $M$ is recessed in the free extremity of end-effector. An articulated chain with serial architectures links the robot bodies which are manipulated using six rotary joints. These six rotary joints are ensured by DC motors each one of them is placed directly on each link. This plant is an example of a non-linear, under-actuated and multivariable system. It has as inputs the torques generated by the DC motors and as outputs the values of its articulation angles.

With the aim of simplifying its modelling calculation, only the two last bodies of the system are taken into consideration. The first one $C_{1}$ is rigid, whereas the second $C_{2}$ is flexible. This flexible solid have a mass load at its end will be treated as a uniform Euler-Bernoulli beam as shown in Fig. 5.

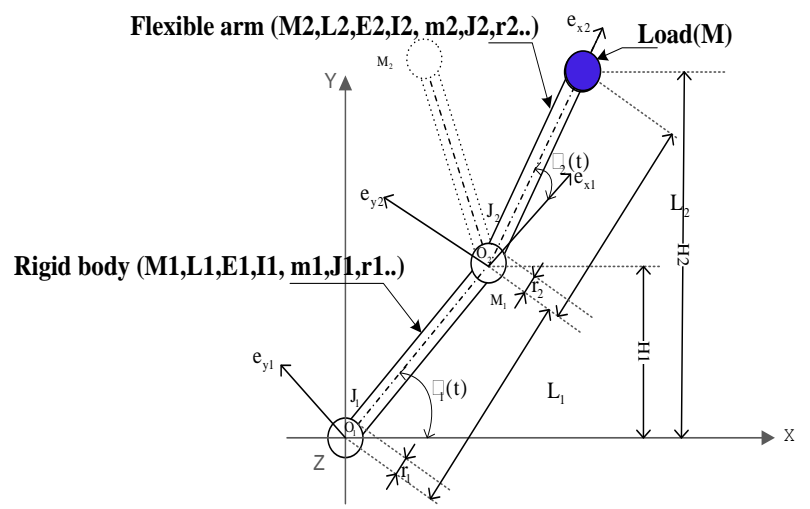

Fig. 5. Two arms of rigid-flexible manipulator robot with its rotary joint.

Where, $M_{i}, L_{i}, I_{i}, E_{i}, m_{i}$ and $l_{i}$ present respectively the mass, the length, the area moment of inertia, the Young's modulus, the mass per length's unit of $i^{\text {th }}$ solid, the length of the $i^{\text {th }}$ element and ${ }^{r_{i}}, J_{i}$ are respectively the radius and the inertia's moment having brought back to the $i^{\text {th }}$ articulation. Moreover, $q_{i}(t)$ presents the rotation angle of the solid $i^{t h}$ around the axis $Z$.

The modelling assumptions will be taking into consideration as follows:
- The robot motion is assumed to be in the vertical plane.

- The shear strain, the effect of the axial force and the rotational inertia of the flexible arm are supposed to be negligible.

- The length of the flexible arm is assumed as constant, in order to avoid the problems appearing from the variability of the flexible arm.

- In order to guarantee the manipulator vibration in the horizontal direction, the depth is considered to be smaller than its length.

\section{B. Dynamic Modelling of the Studied Mechanism}

The dynamic modelling of a rigid-flexible manipulator mechanism provides a description of the relationship between the structure motion and the joint actuators torques [16], [17]. The simulation allows analysing the dynamic performances of the manipulator structure, to test the trajectory planning algorithms and to design the control strategies without the need to use the physical system.

In general, the two most common approaches used to establish the dynamic equation of rigid-flexible manipulator mechanism are the Euler-Lagrange formulation and the Newton-Euler method [18].

The Newton-Euler approach consists of correlating the forces coupling acted on the links displacement and the joint. But, using this method, it is difficult to obtain the dynamic modelling of the robot with several joints. The Euler-Lagrange formulation is described by the energy equilibrium equation which is more adapted in analysing the constraints of the links motion [19].

To develop the dynamic model of the studied mechanism, we will apply besides the Euler-Lagrange approach, the fundamental principles of dynamics and kinematics and the finite elements.

By using the total potential and kinetic energies denoted respectively by $E_{p T o t}$ and $E_{c T o t}$, the Lagrangian is given by

$$
L=E_{c T o t}-E_{p T o t}
$$

According to [20], the Euler-Lagrange equations of motion is described by

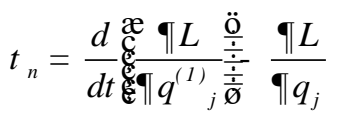

Where, $t_{n}$ states the generalized torques vector at the robot joint, $j$ is body index $(j=1,2)$, the vectors $q_{j}$ and $q^{(1)}$ present, respectively, the joints angles and its velocities. From the Fig. 6, the positions of two points $P_{1}$ and $P_{2}$ placed respectively on the first rigid body and on the second flexible arm are given by

$$
\begin{aligned}
& P_{1}=x_{1} \vec{e}_{x 1} \\
& P_{2}=l_{1} \vec{e}_{x 1}+x_{2} \vec{e}_{x 2}+y_{2} \vec{e}_{y 2}
\end{aligned}
$$


Its derivatives are respectively described as follows:

$$
\begin{aligned}
& V_{1}=x_{1} \dot{\theta}_{1} \vec{e}_{x I} \\
& V_{2}=\left[l_{l} \dot{\theta}_{1} S_{2}-y_{2}\left(\dot{\theta}_{1}+\dot{\theta}_{2}\right)\right] \vec{e}_{x 2}+\left[l_{l} \dot{\theta}_{1} C_{2}+\dot{y}_{2}+x_{2}\left(\dot{\theta}_{1}+\dot{\theta}_{2}\right)\right] \vec{e}_{y 2} \\
& \text { where } S_{2}=\sin \left(\theta_{2}\right) \text { and } C_{2}=\cos \left(\theta_{2}\right)
\end{aligned}
$$

In all expressions, the trigonometric function $\sin \left(q_{i}\right)$ is appointed by $S_{i}$ and the trigonometric function $\cos \left(q_{i}\right)$ is appointed by $C_{i}$.

In our work, it is assumed that the flexible body is divided into two elements and its lateral displacement is described by the B-spline functions [21], [22].

The joint angles and the nodal displacements identify the total degree of freedom of the system presented by

$$
q=\left[\theta_{1}, \theta_{2}, q_{11}, q_{12}, q_{21}, q_{22}\right]^{T}
$$

Where, $q_{11}$ and $q_{21}$ present respectively the nodal displacement related to the first node, $q_{12}$ and $q_{22}$ present respectively the nodal displacement related to the second node.

The calculation of kinetic and potential energies is performed from the elemental energies of each body. For the total kinetic energy $E_{\text {cTot }}$, it is evaluated as the sum of the kinetic energy of the rigid body and that of the flexible body. The general expression can be given by

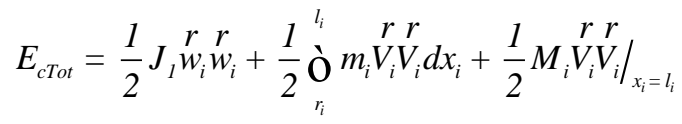

Where, $w_{i}$ is the absolute angular speed of the system and $V_{i}$ is the absolute speed of any point of the $i^{\text {th }}$ body.

The kinetic energy of the rigid body $T_{1}$ can be deduced as follows:

$$
T_{1}=\frac{1}{2}\left(1+f_{I_{l}}\right) I_{R_{l}}+I_{M_{l}} \grave{(}\left(q_{1}^{(1)}\right)^{2}
$$

Where,

$$
\begin{aligned}
& I_{R_{l}}=\frac{1}{3}\left(l_{1}^{3}-r_{1}^{3}\right) m_{l} \\
& I_{M_{l}}=M_{l} I_{l}^{2} \\
& f_{I_{l}}=\frac{J_{1}}{I_{R_{l}}}
\end{aligned}
$$

The flexible body can be presented by uniform EulerBernoulli beam [23]. The kinetic energy can be given by

$$
T_{2}=T_{2 r}+T_{2 f}
$$

Where, $T_{2 r}$ is the kinetic energy of rigid part of the flexible arm and $T_{2 f}$ is the kinetic energy of the flexible part of this arm. The energy $T_{2 r}$ can be deduced as follows:

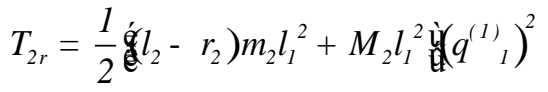

$$
\begin{aligned}
& +\frac{1}{2}\left(1+f_{I_{2}}\right) I_{R_{2}}+I_{M_{2}} \grave{y}\left(q_{1}^{(l)}+q_{2}^{(l)}\right)^{2}
\end{aligned}
$$

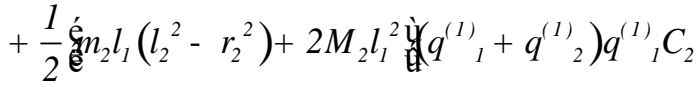

Where,

$$
\begin{aligned}
& I_{R_{2}}=\frac{1}{3}\left(l_{2}^{3}-r_{2}^{3}\right) m_{2} \\
& I_{M_{2}}=M_{2} I_{2}^{2} \\
& f_{I_{2}}=\frac{J_{2}}{I_{R_{2}}}
\end{aligned}
$$

According to the generalized coordinates, the energy $T_{2 f}$ can be deduced as follows:

$$
\begin{aligned}
& T_{2 f}=\frac{1}{2} h^{T} M_{f f} h\left(q^{(1)}{ }_{1}+q^{(1)}{ }_{2}\right)^{2}+\frac{1}{2} h^{(1) T} M_{f f} h^{(1)} \\
& +\left(q^{(1)}{ }_{1}+q^{(1)}{ }_{2}\right) \mathrm{eg}_{f q_{2}} h^{(1)}-M_{f q_{l}} h l_{1} q^{(1)}{ }_{1} S_{2} \text { ̀ํ } \\
& +M_{f q_{l}} h^{(1)} l_{1} q^{(1)}{ }_{1} C_{2}
\end{aligned}
$$

Where, $h$ and $h^{(1)}$ present the vectors of nodal variables of the elementary bodies of the flexible arm and the derivatives and $M_{f f}$ deduced from the Hermite Spline Functions presents the mass matrix of the flexible part.

The total potential energy $E_{p T o t}$ can be composed of the strain energy $U_{a}$ delivered by the centrifugal forces at a point on the Bernoulli's beam, the strain energy $U_{b}$ due to deformation resulted by deflection, the energy $U_{c}$ delivered by the engines and the potential energy $U_{g}$ due to gravity. The energy $U_{a}$ is deduced from

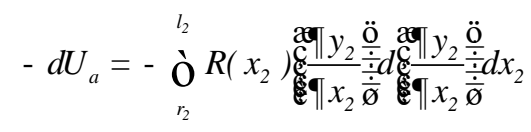

Where, $R\left(x_{2}\right)$ presents the axial load of a point of the Bernoulli beam located at $x_{2}$ and $y_{2}$ is the elementary displacement which can be expressed by

$$
y_{2}(x, t)=\stackrel{a}{i}_{i=1}^{4} f_{i} U_{i}(t)
$$


Where, $\phi_{i}$ is the Hermite Spline functions and $U_{i}(t)$ is the movement (rotation or translation) of resulted by each fictitious joint of the flexible arm [16]. The energy $U_{b}$ can be expressed by

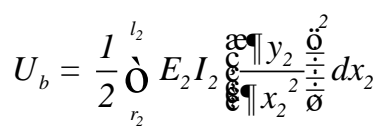

Concerning the energy $U_{c}$, it is described by

$$
U_{c}=-\stackrel{̊}{i}_{i=1}^{2} u_{i} q_{i}
$$

Where, $u_{i}$ is the $i^{\text {th }}$ input torque produced by the $i^{\text {th }}$ actuator. Then, the energy $U_{g}$ is given by

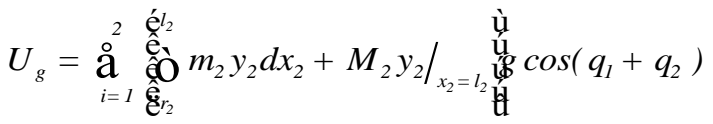

$$
\begin{aligned}
& +\stackrel{\mathrm{a}}{i=1}^{2}\left[m_{i} s_{i} h_{i}\left(q_{1}, q_{2}\right)+M_{i} H_{i}\left(q_{1}, q_{2}\right) g^{r}\right.
\end{aligned}
$$

Where, according to the base, $H_{i}$ presents the $i^{\text {th }}$ body height and $h_{i}$ is that of the gravity centre of the $i^{\text {th }}$ body. Using the previous expressions, the Lagrangian can be deduced with respect to (9). Then, according to the joint variable vector with respect to the time, the derivatives of Lagrangian are deduced. In terms of generalized coordinates, the dynamic equation of the mechanism's model can be calculated and deduced as follows:

$$
\begin{aligned}
& {\left[M+M_{n}(q)+M_{a}(q)\right] q^{(2)}+D_{d} q^{(1)}} \\
& +\ell_{E} K+K_{a}\left(q, q^{(1)}\right) \mathfrak{q}^{q} q+N\left(q, q^{(1)}\right)=B u
\end{aligned}
$$

Where, the sum of the matrices $M$ and $M_{a}(q)$ presents the inertia matrix due to rotation induced by the axial load where the first matrix is linear whereas the second is nonlinear, $M_{n}(q)$ presents the inertia matrix due to the additional rotation delivered by the axial load which is nonlinear, $D_{d}$ presents the damping linear matrix, $K$ presents the generalized geometric stiffness matrix which is nonlinear, $K_{a}\left(q, q^{(1)}\right)$ presents the geometric stiffness matrix due to the rotation induced by the axial load which is linear, $N\left(q, q^{(1)}\right)$ presents the sum of the vector of Gravity's torque and the vector of inertial forces with the second derivatives of the coordinates of the system which is nonlinear, $B$ presents the input matrix and ending with $u$ which presents the input torques delivered by the DC motors.

\section{Linear Parameter Varying Approach for Dynamic Model}

As shown in (26), the dynamic model of the studied system is nonlinear. This nonlinearity caused some problems which can be avoided using the linearization approach. However, this approach is available only in a delimited region and it does not consider the external perturbations. In order to remedy the limits of the linearization method, the LPV approach is applied to the differential equation of the rigidflexible manipulator robot. Several approaches can be used the transformation of a nonlinear dynamic model into an LPV model [24], [25].

The PLV approach is defined as a linear time-varying system presented by its state-space matrices which are given by functions of some varying parameters. It consists of adopting some change of variable methodology which is based on function substitution for the LPV dynamic model of the studied mechanism. In general, the LLV dynamic model can be deduced by continuous-time state-space equations as follows:

$$
\dot{x}(t)=A(\varphi(t)) x(t)+B(\varphi(t)) u(t)
$$

$$
y(t)=C(\varphi(t)) x(t)+D(\varphi(t)) u(t)
$$

Where,

$$
\varphi(t) \in \Theta, \forall t \geq O
$$

Where, $y(t), x(t)$ and $u(t)$ are respectively the outputs, the inputs and the states of the system, $A(\varphi(t)) \in \square^{n_{x} \times n_{x}}$, $B(\varphi(t)) \in \square^{n_{x} \times n_{u}}, C(\varphi(t)) \in \square^{n_{y} \times n_{x}}$ and $D(\varphi(t))$ are timevarying matrices depending to $\varphi(t)$ and $\Theta$ is the polygon of vertices detailing the limit values of the variable parameters.

In our work, the input output and feedforward matrices are invariant that is $B(\varphi(t))=B_{t o t} \quad C(\varphi(t))=C_{t o t} \quad$ and $D(\varphi(t))=O$ can be considered. So, the particular form of LPV system of our model can be shortly presented as follows:

$$
\begin{aligned}
& \dot{x}(t)=A_{l p v}(\varphi(t)) x(t)+B_{t o t} u(t) \\
& y(t)=C_{t o t} x(t)
\end{aligned}
$$

Where, $A_{l p v}$ presents matrix which depends on timevarying parameters given by

$$
A_{l p v}=\left[\begin{array}{cccc}
O & O & 1 & 1 \\
O & O & 1 & 1 \\
O & O & \varphi_{1}(t) & \varphi_{2}(t) \\
O & O & \varphi_{3}(t) & \varphi_{4}(t)
\end{array}\right]
$$

Each variable parameter of the vector $\varphi_{i}(t)$ and its timederivative vary between known extremal values: $\varphi_{i}(t) \in\left[\underline{\varphi}_{i}, \bar{\varphi}_{i}\right]$ and $\dot{\varphi}_{i}(t) \in\left[\underline{\dot{\varphi}}_{i}, \overline{\dot{\varphi}}_{i}\right]$.

\section{NUMERICAL SIMULATIONS AND DISCUSSION}

The rigid-flexible manipulator robot is installed in the laboratory of robotics in the University Polytechnic of Catalonia (UPC) in Spain presented as follows in Fig. 6. 
After obtaining the LPV dynamic model, for several motions profiles, the torque will be applied to each joint of the robot. Table 1 summarizes the numerical values of parameters of the studied mechanism.

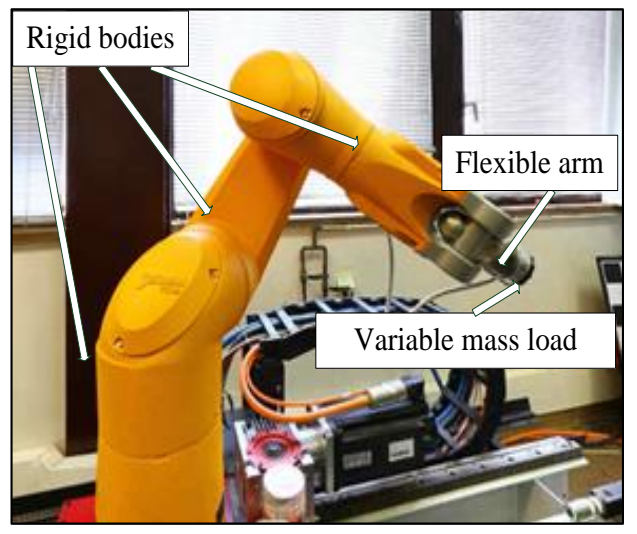

Fig. 6. Rigid-flexible manipulator robot.

TABLE I. DYAMIC PARAMETERS

\begin{tabular}{|l|l|l|}
\hline Parameter & Unit & Value \\
\hline $\mathrm{E}_{1}$ & $\mathrm{GPa}$ & 71 \\
\hline $\mathrm{E}_{2}$ & $\mathrm{GPa}$ & 196 \\
\hline $\mathrm{I}_{1}=\mathrm{I}_{2}$ & $\mathrm{~m}^{4}$ & $1.6710^{-12}$ \\
\hline $\mathrm{m}_{1}=\mathrm{m}_{2}$ & $\mathrm{Kg} / \mathrm{m}$ & 0.831 \\
\hline $\mathrm{r}_{1}=\mathrm{r}_{2}$ & $\mathrm{~m}$ & 0.05 \\
\hline $\mathrm{J}_{1}=\mathrm{J}_{2}$ & $\mathrm{Kg} \cdot \mathrm{m}^{2} / \mathrm{rad}$ & 0.05 \\
\hline $\mathrm{L}_{1}=\mathrm{L}_{2}$ & $\mathrm{~m}$ & 0.375 \\
\hline $\mathrm{M}_{1}$ & $\mathrm{Kg}$ & 0.05 \\
\hline $\mathrm{M}_{2}$ & $\mathrm{Kg}$ & $(0.05)^{2}$ \\
\hline $\mathrm{M}$ & $\mathrm{Kg}$ & 0.01 \\
\hline
\end{tabular}

In our work, the perturbation is presented by the mass load variation and the initial value of this variable is announced in the previous table.

In order to illustrate the validity of the LPV dynamic model of the rigid-flexible manipulator system and effectiveness of the motion profiles in presence of the parameter perturbation, we perform the simulation in MATLAB using a PID controller considering the parameters of Table 2. The parameters of PID controller are chosen by varying manually and gradually its values in order to obtain the best tracking of the robot at the reference.

TABLE II. PID CONTROLLER PARAMETERS

\begin{tabular}{|l|l|l|}
\hline Parameter & Unit & Unit \\
\hline $\mathrm{K}_{\mathrm{P} 1}$ & - & 500 \\
\hline $\mathrm{K}_{\mathrm{I} 1}$ & - & 1 \\
\hline $\mathrm{K}_{\mathrm{D} 1}$ & - & $10^{-3}$ \\
\hline $\mathrm{N}_{1}$ & - & 50 \\
\hline
\end{tabular}

D. With Trapezoidal Profile as Input

In the first case, let apply the trapezoidal motion profile as input to the redundant manipulator robot then the mass load is increased in order to evaluate the system behaviour in presence of disturbance as shown in Fig. 7. We can note that the input discontinuity in one hand and in the other hand the mass load variation has an important impact on the studied system, especially on its flexible structure.

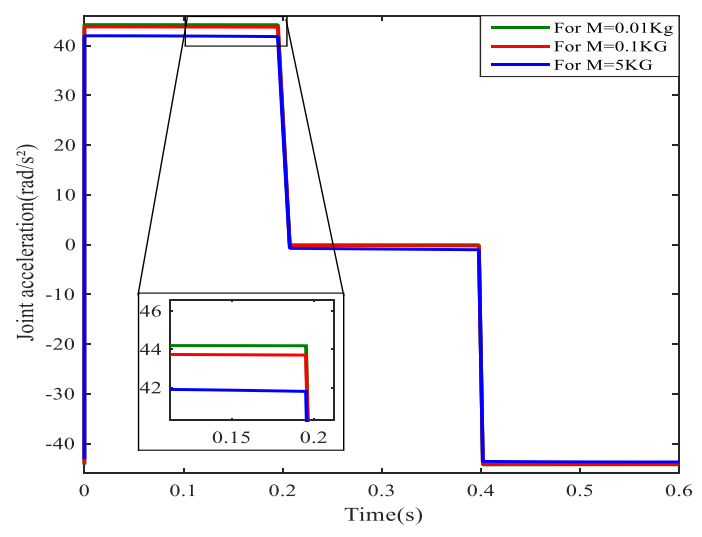

Fig. 7. Torque for trapezoidal profile applied to each joint.

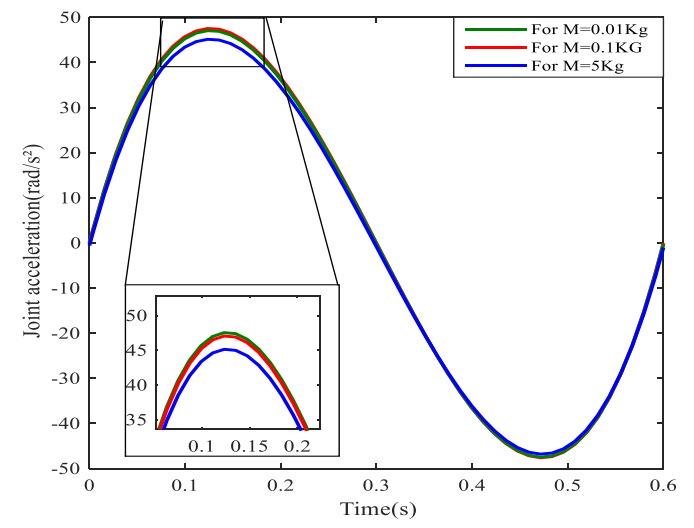

Fig. 8. Torque for fifth-degree polynomial profile applied to each joint.

\section{E. With Fifth-Degree Polynomial Profile as Input}

In another case, a fifth-degree polynomial profile is applied as input along the time period of movement of 0.6 seconds. Fig. 8 shows that this profile keeps the continuity in form of this motion however it does not retain its sign.

With a variable mass load $M$, it is clear that increasing $M$ lead to a higher difference between input and system response as $D_{a}$ increases. From this, it can be deduced that a variation of $M$ and $D_{a}$ is proportional.

\section{F. With Eighth-Degree Polynomial Profile as Input}

Using the same period time of the previous case, the eighth-degree polynomial profile is used to test the system performances in presence of disturbance described by a variable mass load (Fig. 9).

This profile is characterized by the parameter $I_{n d}$ which allows having the smoothest trajectory. In our work, this smoothest trajectory is maintained in $I_{n d}=1.452$.

After applying several tasks with variable mass load, more the mass load increases more the error between the desired trajectory and the system output increases. 


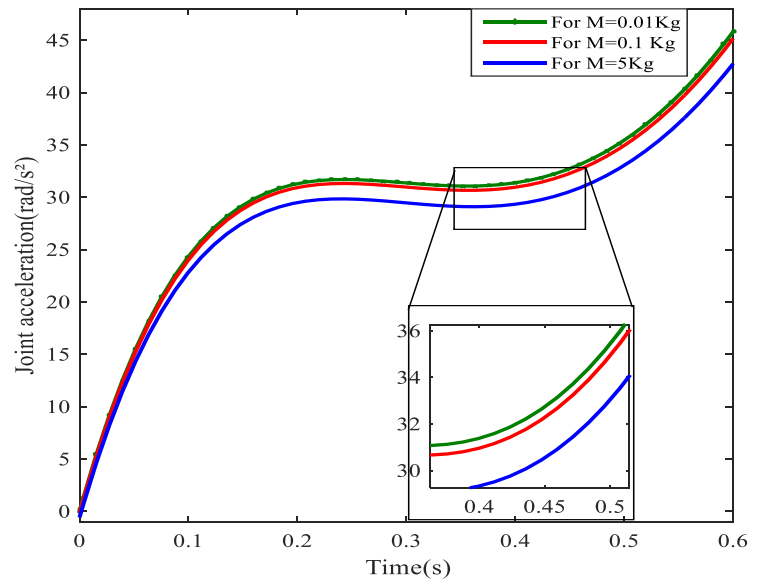

Fig. 9. Torque for eighth-degree polynomial profile applied to each joint.

\section{CONCLUSION}

This paper focuses on the simulation of a redundant manipulator robot. For this purpose, this robot is dynamically modelled using the LPV approach by applying several motions profiles. These inputs are characterized by a continuous torque in minimum time.

The contribution of this paper relies on evaluating the system behaviour by adding to the external perturbation created by the flexible structure of its second arm an external vibration provided by the discontinuous torque applied to the system in a first test and then adding a smooth torque as a motion profile. An improvement in the system performance is obtained through the choice of motion profile characterized by the torque continuity and the movement smoothness.

The results with several tasks demonstrate the effectiveness of the smooth motions profiles and the robustness of LPV modelling using variable mass load as an external distribution.

The future work will be interested in the synthesis of a command that takes into account the flexibility structure of the robot on the one hand and on the other by the input smoothness.

\section{REFERENCES}

[1] X. Yang and S. S. Ge, "Dynamics and tracking control for a free-flying space robot with rigid-flexible links", International Conference on Information and Automation, pp. 394-399, China, 2014.

[2] E. T. S. Alotaibi, H. Al-Rawi, “ Multi-robot path-planning problem for a heavy traffic control application: a servey", Internaional Journal of Advanced Computer Science and Applications, Vol.7, No.6, 2016.

[3] B. Tarvirdizadeh and K. Alipour, "Trajectory optimization of two-link rigid flexible manipulators in dynamic object manipulation missions", $3^{\text {rd }}$ International Conference on Robotics and Mechatronics, pp. 493498, Iran, 2015.

[4] B. Siciliano, L. Sciavicco, L. Villani and G. Oriolo, "Robotics: modelling, planning and control", Advanced Textbooks in Control and Signal Processing, p. 231, 2009.

[5] K. Su, Y. Wang and X. Hu, "Robot path planning based on random coding Particle Swarm Optimization", International Journal of Advanced Computer Science and Applications, Vol.6, No.4, 2015.

[6] D. Gurdan, J. Stumpf, M. Achtelik, K. M. Doth, G. Hirzinger and D. Rus, "Energy-efficient autonomous four-rotor flying robot controlled at
$1 \mathrm{kHz}$, IEEE International Conference on Robotics and Automation, pp. 361-366, Italy, 2007.

[7] K. Khorasani, "Adaptive control of flexible-joint robots", IEEE Transactions On Robotics and Automation, Vol. 8, No. 2, 1992.

[8] T. Huang, P. F. Wang, J. P. Mei, X. M. Zhao, and D. G. Chetwynd, "Time minimum trajectory planning of a 2-dof translational parallel robot for pick-and place operations", CIRP Annals - Manufacturing Technology,Vol. 56, No. 1, pp. 365-368, 2007.

[9] S. Zribi, M. Mejerbi, H. Tlijani and J. Knani, "Comparison between motions profiles applied to flexible manipulator arm", $3^{\text {rd }}$ International Conference on Automation, Control Engineering and Computer Science, pp. 565-671, Tunisia, 2016.

[10] R. Shieh and Y.-S. Lu, "Jerk-constrained time-optimal control of a positioning servo", International Conference on Control, Automation and Systems, in Kintex, Gyeonggi-do, Korea, 2010.

[11] W. P. Bailón, E. B. Cardiel, I. J. Campos and A. R. Paz, "Mechanical energy optimization in trajectory planning for six DOF robot manipulators based on eighth-degree polynomial functions and a genetic algorithm", $7^{\text {th }}$ International Conference on Electrical Engineering, Computing Science and Automatic Control (CCE 2010), pp. 446-451, Chiapas, México, 2010.

[12] H. Hermes and J. P. Lasalle, "Functional analysis and time optimal control", Academic Press, New York, Vol. 56, 1969.

[13] J.-Y. Dieulot, I. Thimoumi, F. Colas and R. Béarée, "Numerical aspects and performances of trajectory planning methods of flexible axes", International Journal of Computers, Communications \& Control, Vol. 1, No. 4, pp. 35-44, 2006.

[14] I. Juarez Campos, "Sobre las regiones geométricas articulares y su aplicación mediante estrategias evolutivas", Doctoral Thesis (In Spanish), Universidad Autónoma de México, 2002.

[15] S. Zribi, H. Tlijani, J. Knani and V. Puig, "On the dynamic modeling and simulation of rigid flexible manipulator robot using several inputs", $4^{\text {th }}$ International Conference on Control, Decision and Information Technologies, in press.

[16] L. Sciavicco and B. Siciliano, "Modeling and control of robot manipulators", $2^{\text {nd }}$ edition, Springer-Verlag London Limited, 2000.

[17] W. Khalil and E. Dombre, "Modeling, identification and control of robots", Kogan Page Science, London, 2002.

[18] B. Sheng, W. Meng, C. Deng and S. Xie, "Model based kinematic \& dynamic simulation of 6-DOF Upper-Limb rehabilitation robot", AsiaPacific Conference on Intelligent Robot Systems, pp. 21-25, Japon, 2016.

[19] M. Shariati Nia, M. Ghayour and M. Mosayebi, "Optimal trajectory planning of a mobile robot with spatial manipulator for obstacle avoidance", International Conference on Control, Automation and Systems, pp. 314-318, Korea, 2010.

[20] H. Roy Pota, "Finite-element/Lagrange modeling and control of a flexible robot arm", $11^{\text {th }}$ International Federation of Automatic Control. World Congress, Vol. 9, pp. 239-243, Estonia, 1990.

[21] P. Rakprayoon, P. Sanposh and N. Chayopitak, "Adaptive and repetitive controller for robotic manipulators with slowly updating scheme using B-spline shape function", IEEE International Conference on Robotics and Biomimetics (ROBIO), pp. 2330-2335, China, 2011.

[22] L. Biagiotti and C. Melchiorri, "Online trajectory planning and filtering for robotic applications via B-spline smoothing filters", IEEE International Conference on Date on Intelligent Robots and Systems (IROS), pp. 5668-5673, Tokyo, 2013.

[23] J. Knani, "Dynamic modelling of flexible robotic mechanisms and adaptive robust control of trajectory computer simulation-Part I", Applied Mathematical Modelling, Vol. 26, pp. 1113-1124, 2002.

[24] H. Halalchi, E. Laroche and G. B. Luliana, "Flexible-link robot control using a linear parameter varying systems methodology", International Journal of Advanced Robotic Systems, 11, pp.1-12, 2014.

[25] D. Rotondo, F. Nejjari and V. Puig, "Quasi-LPV modeling, identification and control of a twin rotor MIMO system", Control Engineering Practice, Vol. 21, No. 6, pp. 829-846, 2013. 